\title{
Identification of TaPPH-7A haplotypes and development of a molecular marker associated with important agronomic traits in common wheat
}

\author{
Huiyan Wang ${ }^{1}$, Shuguang Wang ${ }^{1}$, Xiaoping Chang ${ }^{2}$, Chenyang Hao ${ }^{2}$, Daizhen Sun ${ }^{1^{*}}$ (D) and Ruilian Jing ${ }^{2^{*}}$
}

\begin{abstract}
Background: Premature senescence of flag leaf severely affects wheat yield and quality. Chlorophyll (Chl) degradation is the most obvious symptom during leaf senescence and catalyzed by a series of enzymes. Pheophytin pheophorbide hydrolase (Pheophytinase, PPH) gene encodes a Chl degradation hydrolase.

Results: In this study, the coding, genomic and promoter sequences of wheat TaPPH-A gene were cloned. The corresponding lengths were $1467 \mathrm{bp}, 4479 \mathrm{bp}$ and $3666 \mathrm{bp}$, respectively. Sequence structure analysis showed that TaPPH-A contained five exons and four introns. After the multiple sequences alignment of TaPPH-A genome from 36 accessions in a wheat diversity panel, four SNPs and one 2-bp InDel were observed, which formed two haplotypes, TaPPH-7A-1 and TaPPH-7A-2. Based on the SNP at $1299 \mathrm{bp}$ (A/G), a molecular marker TaPPH-7A-dCAPS was developed to distinguish allelic variation (A/G). Using the molecular markers, 13 SSR, and 116 SNP markers, a linkage map of chromosome 7A were integrated. TaPPH-A was mapped on the chromosome region flanked by XwmC9 (0.94 cM) and AX-95634545 (1.04 cM) on 7A in a DH population. Association analysis between TaPPH-7A allelic variation and agronomic traits found that TaPPH-7A was associated with TGW in 11 of 12 environments and Chl content at grainfilling stage under drought stress using Population 1 consisted of 323 accessions. The accessions possessed TaPPH-7A-1 (A) had higher TGW and Chl content than those possessed TaPPH-7A-2 (G), thus TaPPH-7A-1 (A) was a favorable allelic variation. By analyzing the frequency of favorable allelic variation TaPPH-7A-1 (A) in Population 2 with 157 landraces and Population 3 with 348 modern cultivars, we found it increased from pre-1950 (0) to 1960s (54.5\%), then maintained a relatively stable level about 56\% from 1960s to 1990s.
\end{abstract}

Conclusion: These results suggested the favorable allelic variation TaPPH-7A-1 (A) should be valuable in enhancing grain yield by improving the source (chlorophyll content) and sink (the developing grain) simultaneously. Furthermore, the newly developed molecular marker TaPPH-7A-dCAPS could be integrated into a breeding kit of screening high TGW wheat for marker-assisted selection.

Keywords: Gene cloning, Allelic variation, Molecular marker, TGW, Chl content, Wheat

\footnotetext{
* Correspondence: sdz64@126.com; jingruilian@caas.cn

${ }^{1}$ College of Agronomy, Shanxi Agricultural University, Taigu 030801, China

${ }^{2}$ National Key Facility for Crop Gene Resources and Genetic Improvement/

Institute of Crop Sciences, Chinese Academy of Agricultural Sciences, Beijing

100081, China
}

(c) The Author(s). 2019 Open Access This article is distributed under the terms of the Creative Commons Attribution 4.0 International License (http://creativecommons.org/licenses/by/4.0/), which permits unrestricted use, distribution, and reproduction in any medium, provided you give appropriate credit to the original author(s) and the source, provide a link to the Creative Commons license, and indicate if changes were made. The Creative Commons Public Domain Dedication waiver (http://creativecommons.org/publicdomain/zero/1.0/) applies to the data made available in this article, unless otherwise stated. 


\section{Background}

Wheat (Triticum aestivum L.) provides the staple food source for $30 \%$ human population in the world [1]. It is estimated that the global demand for wheat will increase by a further $40 \%$ before 2020 , because of an increasing world population [2]. Therefore, breeding for high-yield varieties has still been a major objective in wheat breeding programs [3]. Thousand-grain weight (TGW) is an important yield-contributing trait. The significantly genetic improvement in wheat grain yield is partially attributed to increased TGW [4].

Delayed senescence, or stay-green, enables leaf to maintain a longer greenness after anthesis, and contributes to a longer grain-filling period [5, 6]. Gregersen, et al. [7] reported a positive correlation between delayed crop senescence and grain yield. A two-days delay in onset of senescence increased 11\% carbon fixed in Lolium temulentum L [8]. Furthermore, the yield of wheat stay-green mutant tasg1 was 9.5\% higher than the wild type [9]. Thus it has been regarded as a desirable characteristic for the production of a number of crops including wheat. Chlorophyll (Chl) degradation is the main indicator of leaf senescence and catalyzed by a series of enzymes [10-14]. Pheophytin pheophorbide hydrolase (Pheophytinase, $\mathrm{PPH})$ is a key enzyme in Chl degradation, specifically hydrolyzes pheophytin a to pheophorbide a $[15,16]$. Mutagenesis or overexpression of $P P H$ can lead to a stay-green or premature senescence phenotype in Arabidopsis and rice $[15,16]$. In addition, overexpressing $L p P P H$ also accelerated Chl degradation, and the expression level was positively related to leaf senescence [17]. Therefore, the expression of $P P H$ gene affected Chl degradation, further affected yield and quality of crops.

Marker-assisted selection is considered a potential approach to accelerate the process of wheat breeding [18, 19]. Single nucleotide polymorphism (SNP) mainly refers to DNA sequence polymorphisms caused by single nucleotide changes and small insertions/deletions [20, 21]. Comparing with other marker types, such as RFLP, RAPD, AFLP, SSR and ISSR, SNP is a kind of more valuable marker due to its high abundance and relative stability, cost efficiency, and high-throughput scoring [22, 23]. Additionally, SNP has been also proved to be a kind of very effective molecular markers in high-density genetic map construction, fine mapping, genetic diversity, association analysis, functional marker development and markerassisted selection breeding $[24,25]$. Along with the development of high density genetic map of SNPs, association analysis has been proved to be an effective tool for identifying the relationship between polymorphic sites of target genes and important quantitative traits, and has been widely performed in many plant species, such as Arabidopsis [26, 27], rice [28, 29], maize [30-32], and wheat $[33,34]$.
The $P P H$ plays an important role during Chl degradation process. But the sequence, structure and polymorphism of TaPPH are still unclear in wheat. The objectives of this study were (i) to isolate the coding, genomic and promoter sequences of TaPPH-A, (ii) to identify polymorphism sites and develop molecular marker(s) from TaPPH$A$, (iii) to identify favorable allelic variation, (iv) to evaluate the value of the newly molecular marker(s) by analyzing the geographic distribution and frequency of favorable allelic variation. The purpose is to provide a newly and effectively molecular marker for breeding high yield wheat variety by marker-assisted selection. The scheme of the whole research work is as follows (Fig. 1).

\section{Results}

Cloning and characterization of TaPPH-A gene

To obtain possible reference sequence of TaPPH gene, the nucleotide sequence of OsNYC3 (Os06g0354700) was used as a query against Chinese Spring genome sequences database in the URGI (https://urgi.versailles. inra.fr/blast/blast.php). Three Chinese Spring wheat scaffold sequences (IWGSC_V3_chr7AL_scaffold_1626, IWGSC_V3_chr7BL_scaffold_507, IWGSC_V3_chr7DL_ scaffold_2847) with high similarity (> 80\%) were selected and identified as potential candidate sequence. The three scaffold sequences were downloaded, and used for nucleotide alignment through SeqMan. A genome-specific primer pair G-F/R and a promoter-specific primer pair $\mathrm{P}-\mathrm{F} / \mathrm{R}$ were designed, and used to clone the genomic and promoter sequences of TaPPH-A in Chang 4738. The corresponding lengths of the amplified fragments were $4125 \mathrm{bp}$ and $4138 \mathrm{bp}$, respectively. Sequence analysis indicated that the genomic and promoter sequences of TaPPH-A were $4479 \mathrm{bp}$ and $3666 \mathrm{bp}$ in length, respectively. To analyze the gene structure of TaPPH- $A$, the coding sequence was cloned using a pair of primers $\mathrm{C}-\mathrm{F} / \mathrm{R}$ with the length of $1467 \mathrm{bp}$. Compared with the coding sequence, the genomic sequence of TaPPH- $A$ consisted of five exons and four introns (Fig. 2a).

\section{Sequence polymorphism and molecular marker development for TaPPH-A}

To detect polymorphisms in the genomic and promoter sequences of TaPPH- $A$, all accessions in wheat diversity panel were used to amplify the TaPPH-A gene, and the amplified fragments were sequenced. Multiple sequence alignment found that four SNPs (G/A, A/G, C/T, A/C) and a 2-bp InDel were observed in the genomic sequence, while no sequence variation was detected in the promoter sequence of TaPPH-A (Fig. 2a and b). From the start codon (ATG), four SNPs were located at $1038 \mathrm{bp}, 1299$ bp, $1468 \mathrm{bp}$, and $3790 \mathrm{bp}$, and the 2-bp InDel was identified from 1902 to $1903 \mathrm{bp}$. The four SNPs and the 2-bp 

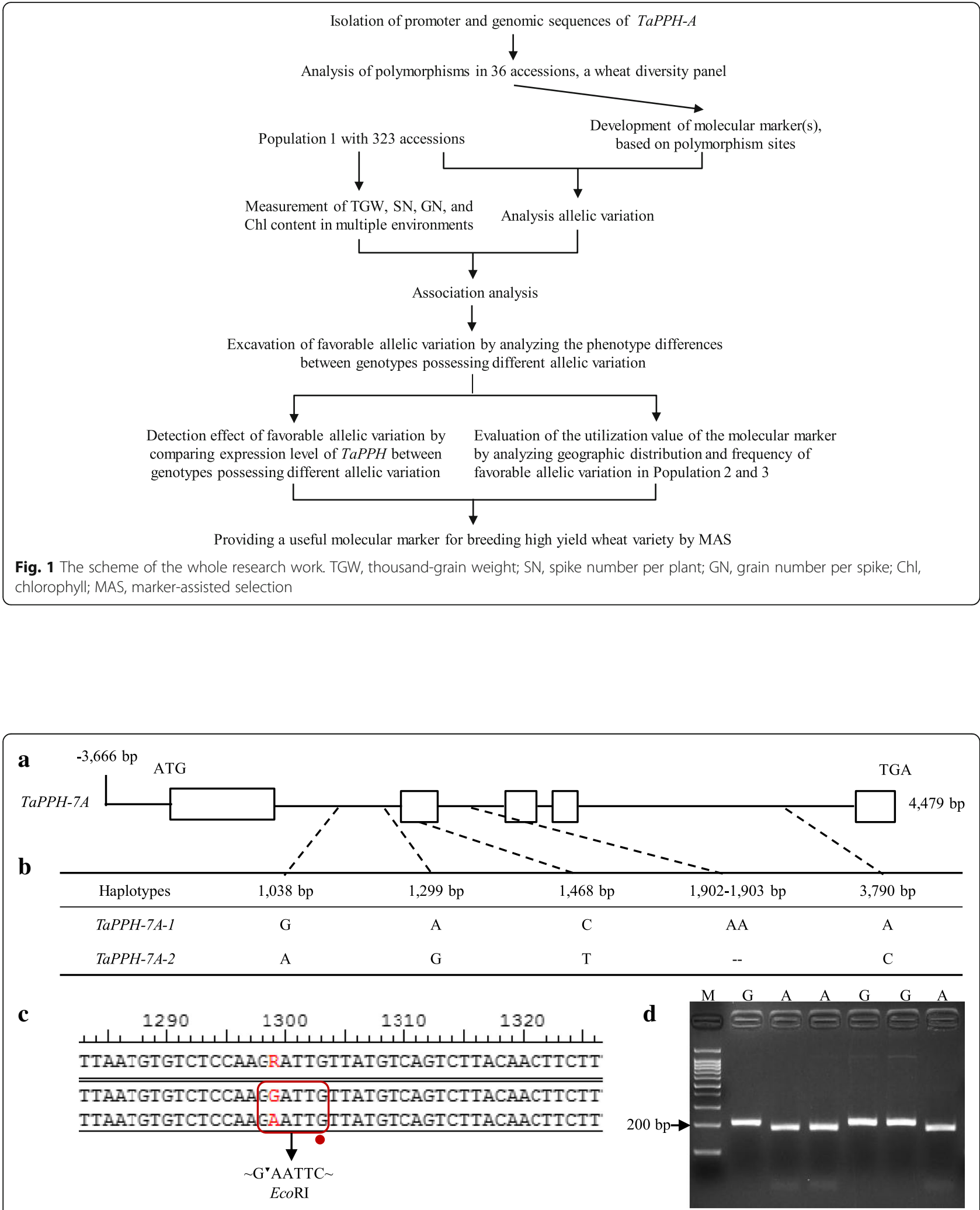

Fig. 2 Single nucleotide polymorphisms and molecular marker of TaPPH-7A. (a) Schematic diagram of TaPPH-7A structure. (b) SNP and InDel in two haplotypes identified in TaPPH-7A among the wheat diversity panel. (c) The molecular marker TaPPH-7A-dCAPS, formed EcoRI restriction site by changing the base $\mathrm{G}$ to $\mathrm{C}$. The EcoRI restriction site and a base $\mathrm{G}$ mismatched to $\mathrm{C}$ are marked in red rectangle and red point, respectively. (d) PCR products were digested by ECoRI. M is a $100 \mathrm{bp}$ DNA Ladder 
InDel together formed two haplotypes, TaPPH-7A-1 and TaPPH-7A-2 (Fig. 2b).

Based on the SNP at $1299 \mathrm{bp}(\mathrm{A} / \mathrm{G})$, a molecular marker was developed and named TaPPH-7A-dCAPS. It contained a base $\mathrm{G}$ mismatched to $\mathrm{C}$ in the downstream primer EcoRI-R and a restriction enzyme EcoRI site (Fig. 2c). To characterize the observed TaPPH-A allelic variation in large wheat populations, the following three experiments were performed: firstly, the genome-specific primer pair, G-F/R, was used to amplify genomic sequence of TaPPH$A$, and a 4125 bp fragment was obtained. Secondly, $0.2 \mu \mathrm{L}$ PCR product of $4125 \mathrm{bp}$ was used as the template to amplify a $202 \mathrm{bp}$ fragment by the molecular marker primer pair, EcoRI-F/R. Thirdly, the PCR product of $202 \mathrm{bp}$ possessed allelic variation TaPPH-7A-1 (A) formed a EcoRI site GAATTC (Fig. 2c), and was digested into 174 bp and $28 \mathrm{bp}$ by restriction enzyme EcoRI (Fig. 2d). Whereas the corresponding PCR product possessed allelic variation TaPPH-7A-2 (G) was GGATTC (Fig. 2c), and corresponding product remained $202 \mathrm{bp}$ (Fig. 2d).

\section{Genetic mapping of TaPPH}

Fifteen wheat species of different ploidy and a set of Chinese Spring nulli-tetrasomic lines were used to assign chromosomal location to TaPPH-A. Target fragment was only detected in accessions possessing chromosomes 7A, including three AA genome accessions, three AABB genome accessions, three AABBDD genome accessions, and all nulli-tetrasomic lines besides N7AT7B and N7AT7D of Chinese Spring (Fig. 3a). This indicated that TaPPH was located on chromosome 7A. To further map its position, the molecular marker, 13 SSR and 116 SNP markers were integrated into a linkage map of chromosome 7A. Using the DH population derived from the cross of Hanxuan $10 \times$ Lumai 14 , TaPPH was mapped on the chromosome region flanked by $X w m c 9(0.94 \mathrm{cM})$ and $A X-95634545$ (1.04 cM) on 7A (Fig. 3b). Therefore, it was named as TaPPH-7A.

\section{Association of TaPPH-7A with yield-related traits}

To detect association of TaPPH-7A allelic variation with yield-related traits, the Population 1 consisted of 323 wheat accessions was used. In Population 1, TaPPH-7A-1 (A) was a major allelic variation, accounting for $69.3 \%$ of the total accession number (Additional file 1: Figure S1). TaPPH-7A had no significant association with GN in all 12 environments, and no significant association with $\mathrm{SN}$ in seven environments (E1, E2, E3, E5, E6, E7, and E10) (Table 1). However, significant association between TaPPH-7A and TGW was observed in 11 of 12 environments, except for E10 (Table 1). Genotypes possessing allelic variation TaPPH-7A-1 (A) had significantly higher TGW than those possessing allelic variation TaPPH7A-2 (G) (Fig. 4a). Thus, TaPPH-7A-1 (A) could be a favorable allelic variation for improving TGW.

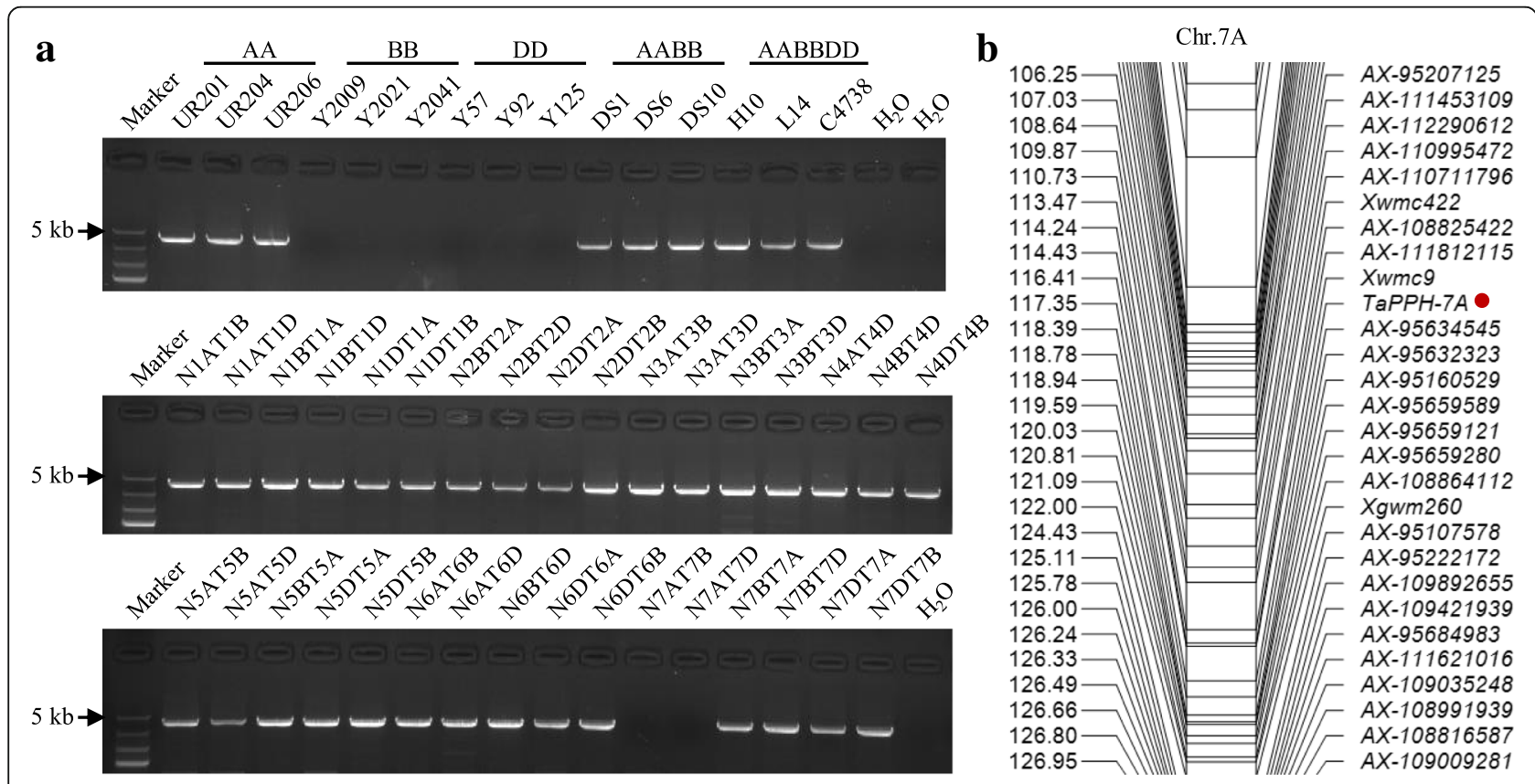

Fig. 3 Chromosome location and genetic mapping of TaPPH. (a) Location of TaPPH on chromosome 7A using 15 wheat species of different ploidy and 33 nulli-tetrasomic lines of Chinese Spring. H10, Hanxuan 10; L14, Lumai 14; C4738, Chang 4738. (b) Linkage map of TaPPH on wheat chromosome 7A flanked by SSR marker XWmC9 and SNP marker AX-95634545 in the DH population. The target location is marked by red point 
Table 1 Association analysis of TaPPH-7A allelic variation and yield-related traits in 12 environments

\begin{tabular}{llll}
\hline Environment & SN (P-value $)$ & GN $(P$-value $)$ & TGW $(P$-value $)$ \\
\hline E1 & 0.11 & 0.65 & $8.65 \mathrm{E}-04^{* *}$ \\
E2 & 0.91 & 0.15 & $3.56 \mathrm{E}-04^{* *}$ \\
E3 & 0.84 & 0.39 & $0.01^{*}$ \\
E4 & $4.56 \mathrm{E}-04^{* *}$ & 0.13 & $0.02^{*}$ \\
E5 & 0.83 & 0.36 & $3.10 \mathrm{E}-03^{* *}$ \\
E6 & 0.69 & 0.92 & $7.60 \mathrm{E}-05^{* *}$ \\
E7 & 0.15 & 0.80 & $1.90 \mathrm{E}-03^{* *}$ \\
E8 & $2.50 \mathrm{E}-03^{* *}$ & 0.13 & $2.32 \mathrm{E}-04^{* *}$ \\
E9 & $6.14 \mathrm{E}-04^{* *}$ & 0.86 & $0.044^{*}$ \\
E10 & 0.26 & 0.07 & 0.41 \\
E11 & $0.02^{*}$ & 0.36 & $4.97 \mathrm{E}-04^{* *}$ \\
E12 & $3.39 \mathrm{E}-04^{* *}$ & 0.33 & $8.43 \mathrm{E}-04^{* *}$ \\
\hline
\end{tabular}

SN, spike number per plant; GN, grain number per spike; TGW, thousand-grain weight; E1 to E12, the environments of 2015-SY-WW, 2015-SY-DS, 2015-SYWW + HS, 2015-SY-DS + HS, 2016-CP-WW, 2016-CP-DS, 2016-SY-WW, 2016-SYDS, 2016-SY-WW + HS, 2016-SY-DS + HS, 2017-SY-WW and 2017-SY-DS, respectively. SY, Shunyi; CP, Changping; WW, Well-watered; DS, Drought stress; HS, Heat stress. ${ }^{*} P<0.05$ and ${ }^{* * *} P<0.01$, respectively

\section{Association of $\mathrm{TaPPH}-7 \mathrm{~A}$ with $\mathrm{Chl}$ content}

In Population 1, we also found that TaPPH-7A had a significant association with $\mathrm{Chl}$ content at grain-filling stage under drought stress (Table 2). Genotypes possessing allelic variation TaPPH-7A-1 (A) had significantly higher Chl content than those of possessing allelic variation TaPPH-7A-2 (G) (Fig. 4b). So TaPPH-7A-1 (A) might be a
Table 2 Association analysis of TaPPH-7A allelic variation and Chl contents in two environments

\begin{tabular}{|c|c|}
\hline Environment & Chl ( $P$-value) \\
\hline$\overline{E 11-F}$ & 0.30 \\
\hline E11-GF & 0.22 \\
\hline E12-F & 0.49 \\
\hline E12-GF & $1.46 \mathrm{E}-04^{* *}$ \\
\hline
\end{tabular}

superior allelic variation to delay Chl degradation at grainfilling stage under drought stress.

\section{Quantitative real-time PCR analysis of TaPPH-7A expression}

To detect effect of favorable allelic variation on TGW and Chl content, six accessions each possessing TaPPH7A-1 (A) and TaPPH-7A-2 (G) were randomly selected from Population 1, and used to test the expression level of TaPPH-7A (Additional file 3: Table S1). The melting curves of qRT-PCR for genes TaPPH-7A and TaActin were both single peaks, indicating primer specificity of the amplification (Additional file 2: Figure S2). qRT-PCR revealed that genotypes with TaPPH-7A-1 (A) had lower relative expression level than those with TaPPH-7A-2 (G) (Fig. 5). This finding suggested that lower relative expression level of TaPPH-7A-1 might help to maintain a higher $\mathrm{Chl}$ content, thus synthesizing more photosynthates, furthermore improving TGW.

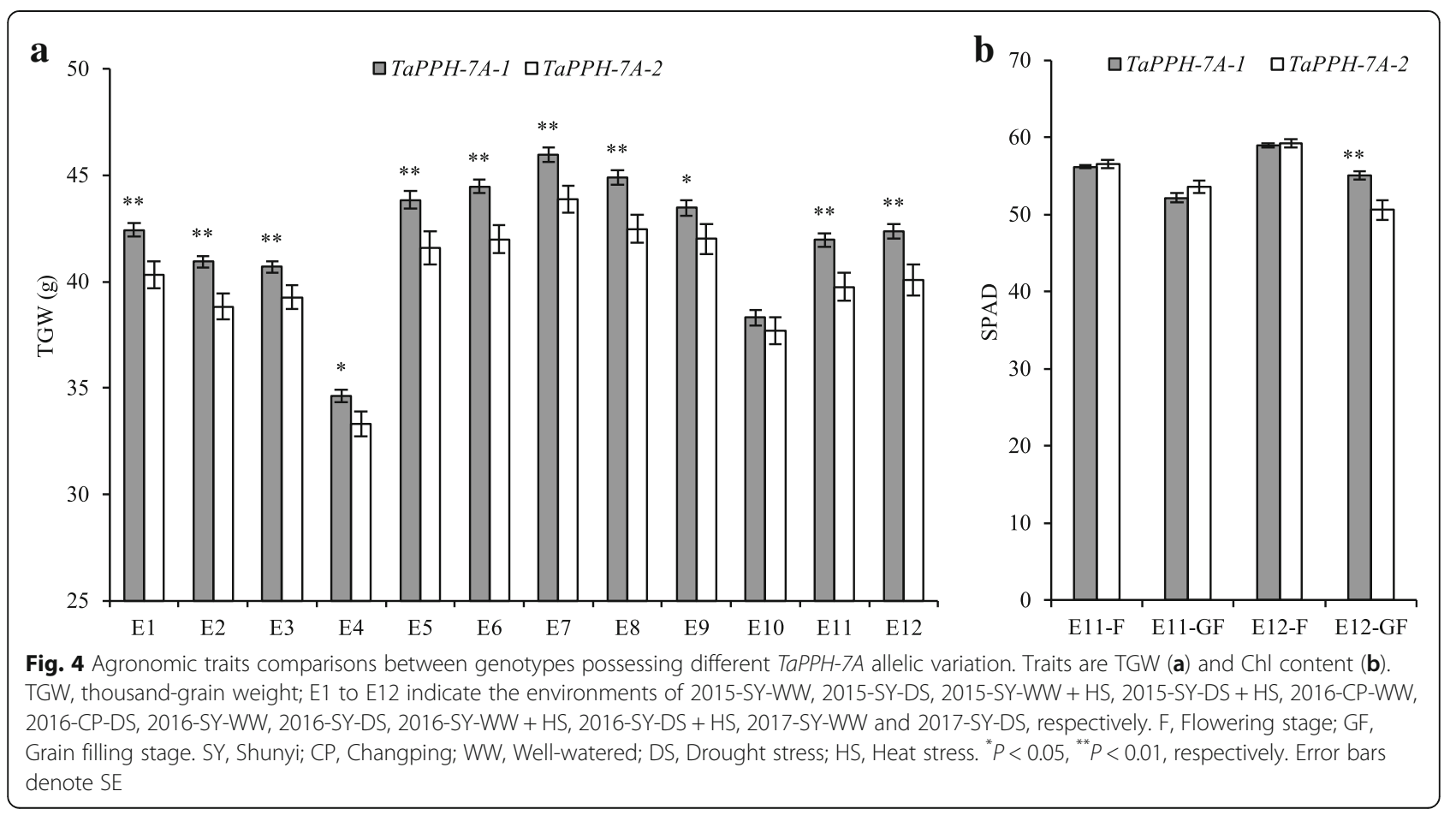




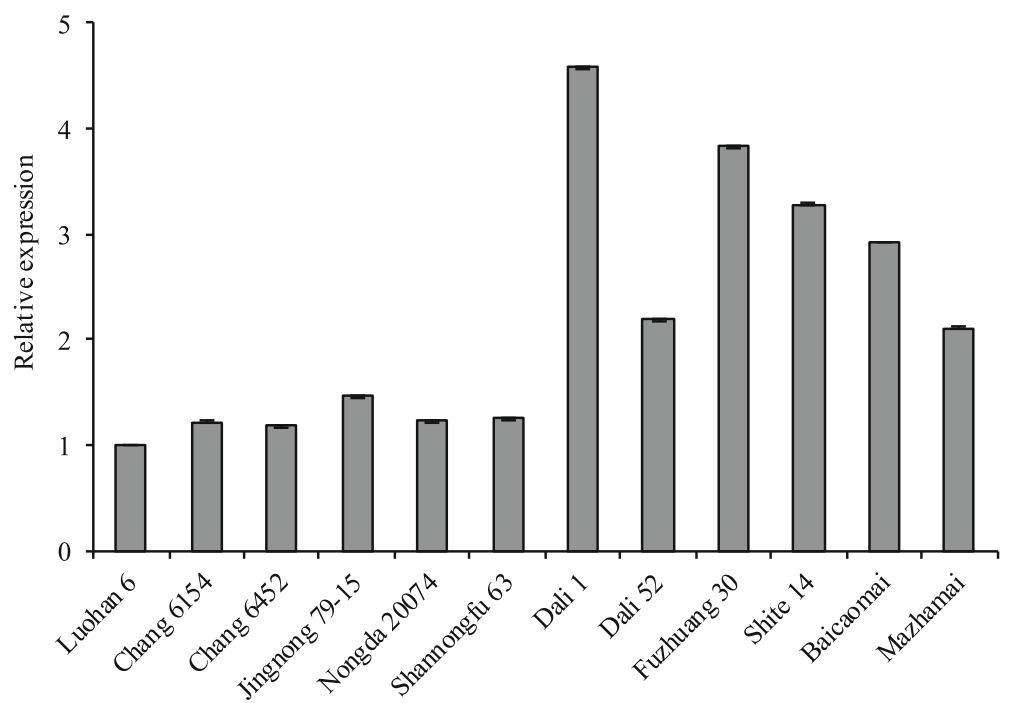

Fig. 5 Relative expression levels of two TaPPH-7A allelic variation in leaves at grain filling stage. The first six genotypes, Luohan 6, Chang 6154, Chang 6452, Jingnong 79-15, Nongda 20,074, and Shannongfu 63 possess the allelic variation TaPPH-7A-1 (A). The second six genotypes, Dali 1 , Dali 52, Fuzhuang 30, Shite 14, Baicaomai, and Mazhamai possess the allelic variation TaPPH-7A-2 (G). Error bars denote SE

\section{Geographic distribution of TaPPH-7A allelic variation in Chinese wheat production zones}

In China, ten wheat Zones were divided according to wheat types, growing seasons, varietal response to photoperiod, temperature, moisture, biotic and abiotic stresses [35]. In this study, all accessions of Population 2 with 157 landraces and Population 3 with 348 modern cultivars were collected from the ten wheat Zones (Additional file 4: Table S2). The geographic distribution of TaPPH-7A allelic variation was evaluated using Population 2 and Population 3. In Population 2, only 12 accessions (7.6\%) possessed favorable allelic variation TaPPH-7A-1 (A), and the frequency was much lower than that of TaPPH-7A-2 (G) (Additional file 1: Figure S1). The largest frequency of TaPPH-7A-1 (A) was $30.0 \%$ in Zone X, followed by $16.7 \%$ in Zones V and VI. In Population 3, favorable allelic variation TaPPH-7A-1 (A) was identified in 183 accessions (52.6\%) (Additional file 1: Figure S1), and the frequencies in all ten wheat Zones were higher than those in Population 2. In Zones I, II, III and VI, which accounted for about $75 \%$ China wheat area, the frequencies of TaPPH-7A-1 (A) were higher than that of TaPPH-7A-2 (G), but in other wheat growing areas, such as Zones V, VIII and $\mathrm{X}$, the frequencies of TaPPH-7A-1 (A) were obviously lower than TaPPH-7A-2 (G) (Additional file 4: Table S2, Fig. $6 \mathrm{a}$ and $\mathrm{b})$. Thus, favorable allelic variation TaPPH-7A-1 (A) was strongly selected in the major wheat production regions, and weakly selected in other wheat production regions.

\section{The frequency of $T a P P H-7 A$ allelic variation in wheat breeding history}

To evaluate the frequency of favorable allelic variation TaPPH-7A-1 (A), 334 accessions known released dates from Population 3 were used and divided into six groups (pre-1950, 1950s, 1960s, 1970s, 1980s and 1990s). All eight accessions, which released before 1950 (pre-1950), possessed the allelic variation TaPPH-7A-2 (G). The frequency of TaPPH-7A-1 (A) increased from pre-1950 (0) to $1960 \mathrm{~s}(54.5 \%)$, then maintained a relatively stable level about $56 \%$ from 1960 s to 1990 s (Fig. 7). The TGW exhibited a trend of continuous increase from pre-1950 to 1990 s (Fig. 7). These strongly indicated that favorable allelic variation TaPPH-7A-1 (A) should be valuable, and could be selected to improve TGW.

\section{Discussion}

\section{Sequence polymorphism of $\mathrm{TaPPH}-7 \mathrm{~A}$}

During the evolution, domestication and breeding in wheat, the genetic information had undergone two doublings and mutations under natural conditions, which eventually resulted in a rich genetic diversity. Somers, et al. [36] reported the level of sequence polymorphism was 1 SNP every 540 bp of EST sequence using a bioinformatics strategy based on a large wheat EST database from 12 cultivars. Ravel, et al. [37] identified that the SNP frequency was $1 \mathrm{SNP} / 334 \mathrm{bp}$ in the genomic sequence including coding and non-coding region, and $1 \mathrm{SNP} / 267 \mathrm{bp}$ in coding region in wheat. In this study, polymorphisms of TaPPH-7A gene sequences from the wheat diversity panel (36 accessions) was detected by direct sequencing. The SNPs occurred in both exons and introns region of TaPPH-7A (Fig. 2). This study allowed us to estimate an average of 1 SNP for every $1120 \mathrm{bp}$ in the genomic sequence including coding and non-coding region, and 1 SNP every 1467 bp in coding region, 1 SNP every 1004 bp 


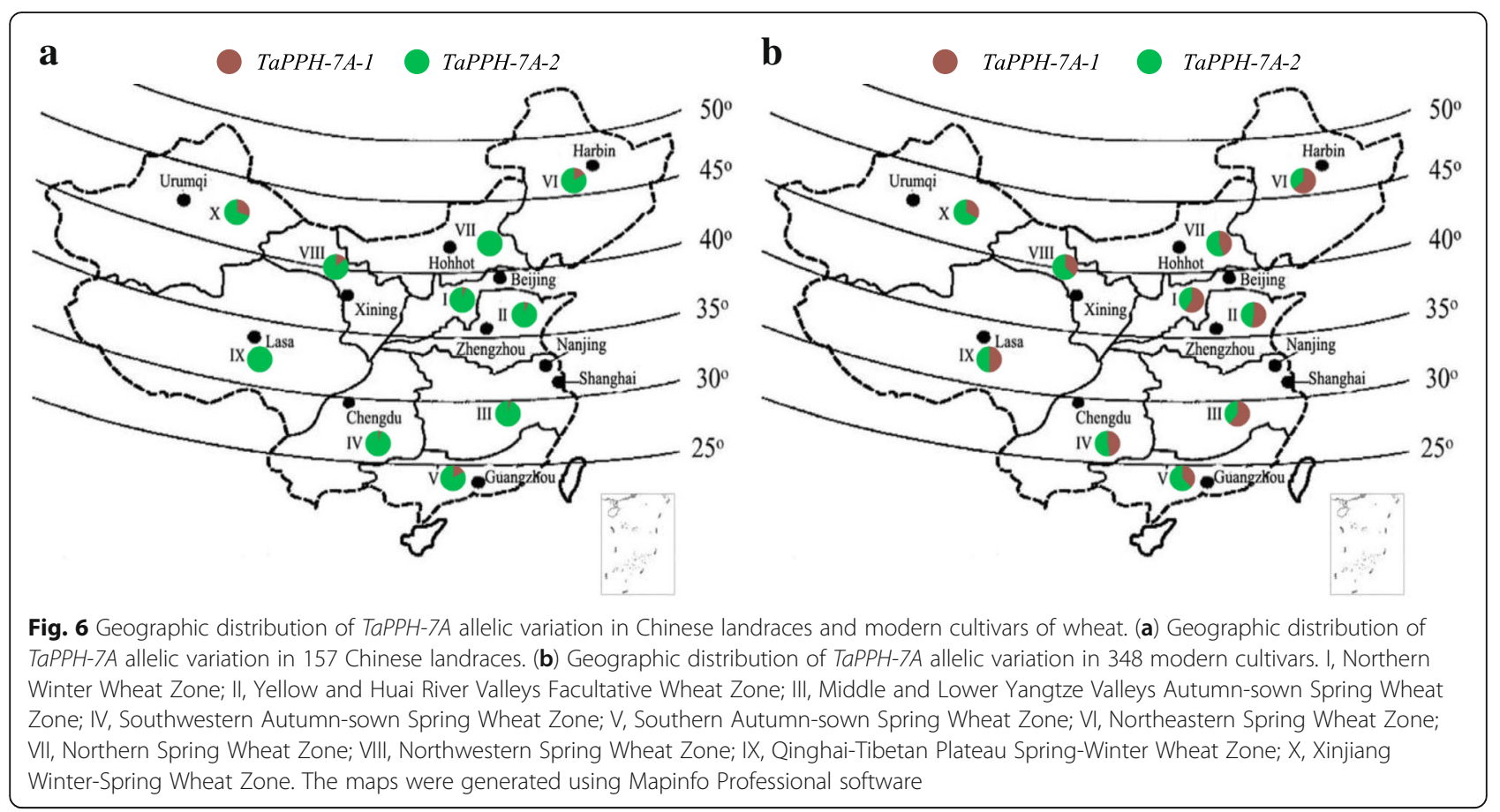

in non-coding region, but no polymorphism detected in the promoter region. As expected, the coding region had lower SNP frequency than the non-coding region, and this result is consistent with earlier studies [38]. However, the SNP frequency of the whole genomic sequence was much lower than those in the previous studies [36, 37]. This result suggested that TaPPH-7A is a relatively conservative gene in evolution.

In this study, only one synonymous mutation SNP (C/ $\mathrm{T})$ was identified in the exon region at $1468 \mathrm{bp}$ of
TaPPH-7A. However, the TaPPH-7A associated with TGW and Chl content, and the relative expression levels of $\mathrm{TaPPH}-7 \mathrm{~A}$ were lower in wheat genotypes with TaPPH-7A-1 (A) than those with TaPPH-7A-2 (G) (Fig. $5)$, so the reason for the phenotypic and gene expression variation may be alternative splicing. Many previous studies had showed that variations in the introns can significantly affect gene expression or phenotypic variation by alternative splicing of pre-mRNA [39-42]. Thus, variations in introns are important and worthy of future investigation.

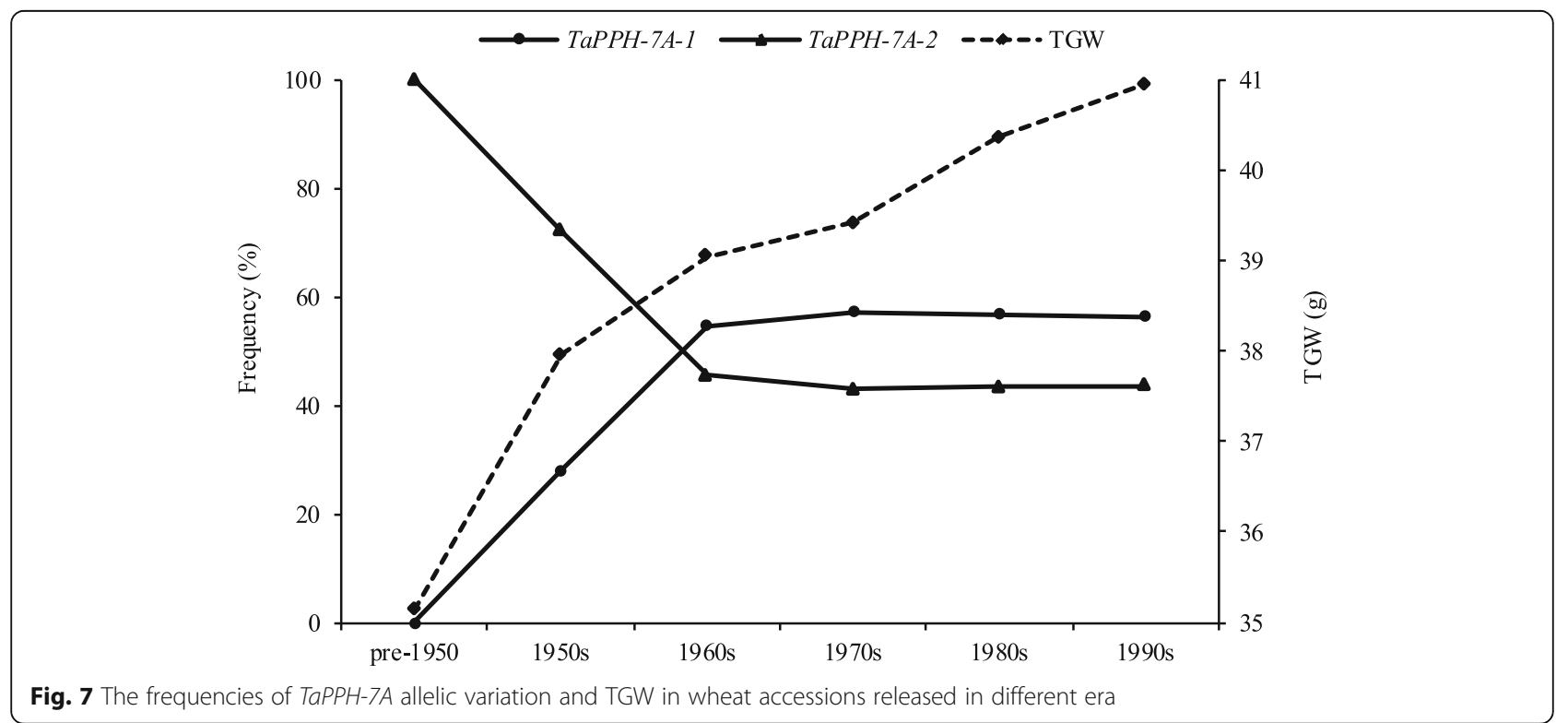


TaPPH-7A is a novel locus related to TGW and Chl content Using QTL mapping, it is possible to dissect loci controlling genetic variation and characterize these loci based on map position, phenotypic effects, gene actions, pleiotropic effects, and epistatic interactions with other QTL in a segregating population [43]. Several QTL for Chl content and TGW were detected on wheat chromosome $7 \mathrm{~A}$ in various mapping populations. Using the $\mathrm{DH}$ population derived from the cross of Hanxuan $10 \times$ Lumai 14, Shi, et al. [44] found two QTL for Chl content at flowering and grainfilling stage on chromosome 7A with 7.32 and $8.36 \%$ of phenotypic variation, respectively, and Yang, et al. [45] detected a QTL for Chl content in the interval Xwmc488P2071-180 on chromosome 7A under water deficient. Bhusal, et al. [46] also reported a QTL for Chl $a$ content on chromosome 7A close to the marker Xwmc388 under heat stress. Ilyas, et al. [47] mapped a QTL for total Chl content on 7A flanked by Xbarc49 and Xgdm14. As for TGW, a few QTL have been reported on chromosome 7AL in wheat, too. For example, Wang, et al. [48] reported a QTL for TGW close to the marker Xcfa2257 in a natural population, which explained up to $21.99 \%$ of the total phenotypic variation. Groos, et al. [49] also found a QTL for TGW in the vicinity of Xgwm282, explaining phenotypic variation ranging from 5.2 to $10.3 \%$ in six environments. In the present study, $\mathrm{TaPPH}$ was located on chromosome 7A between markers $X w m c 9$ and $A X-95634545$ within an interval of $1.98 \mathrm{cM}$ by QTL mapping (Fig. 3b). Based on the common wheat consensus maps [50, 51], TaPPH-7A is considered to be near to the centromere, but is far from markers Xwmc488 and P2071-180 [45], Xwmc388 [46], Xbarc49 and Xgdm14 [47], Xcfa2257 [48], and Xgwm282 [49]. Thus, TaPPH-7A was likely a novel locus related to Chl content and TGW on chromosome 7A.

\section{TaPPH-7A-dCAPS is a stably and effectively molecular marker for assisted selection breeding}

Functional molecular marker was developed from polymorphic site within gene causally affecting phenotypic trait variation [52]. Converting SNPs to CAPS or dCAPS markers enable SNPs to be more conveniently applied in selecting preferred alleles in marker-assisted breeding [18]. In addition, the assay procedure of CAPS or dCAPS marker was simple. However, it is difficult to develop CAPS or dCAPS markers from identified wheat genes because of the large allohexaploid genome [53, 54]. In order to solve this problem, we firstly designed genome-specific primer to distinguish three ortholog genome sequences, then identified SNPs by comparing the genomic sequences of target gene from different wheat genotypes, finally developed molecular markers based on SNP. Using the method described above, a molecular marker TaPPH-7A-dCAPS was developed based on the SNP at 1299 bp in this study (Fig. 2c). Two allelic variation, TaPPH-7A-1 (A) and TaPPH-7A-2 (G), were detected by scanning the Population 1 using the developed molecular marker (Additional file 5: Table S3). We made further efforts to found that favorable allelic variation TaPPH-7A-1 (A) was associated with high TGW in 11 of 12 environments and $\mathrm{Chl}$ content at grain-filling stage under drought stress using Population 1 (Fig. 4). And we also found that genotypes with favorable allelic variation TaPPH-7A-1 (A) had lower relative expression level of TaPPH-7A than those with TaPPH-7A-2 (G) (Fig. 5). Therefore, it is deduced that the favorable allelic variation, TaPPH-7A-1 (A), could simultaneously improve the source (chlorophyll content) and sink (the developing grain) after flowering, and eventually contribute to wheat grain weight. Thus, the newly developed molecular marker, TaPPH-7A-dCAPS, is a stable and effectively molecular marker for grain yield, and can be used to marker-assisted selection breeding in wheat.

\section{Favorable allelic variation TaPPH-7A-1 (a) was selected in wheat breeding history}

Wheat is one of the most important staple food crops in China. With the development of economy, the breeding objective is constantly changing. Before 1960s, the main breeding objective was to increase TGW and further improve yield. From 1970s to 1990s, the breeding objective was changed to improve agronomic traits such as plant height, quality, spike number and grain weight per spike [55]. The change of Chinese wheat breeding objective from 1950 to 1960 s resulted in the positive selection of favorable allelic variation TaPPH-7A-1 (A) and the rapid increase in TGW before 1970 (Fig. 7). From 1970s to 1990s, the frequency of favorable allelic variation TaPPH-7A-1 (A) maintained a relatively stable level about 56\%, but TGW continuously increased (Fig. 7), the possible reason may be that other genes contributing to TGW were selected, such as TaSnRK2.3-1A (Hap-1A1) and TaSnRK2.3-1B (Hap-1B-1) [56], TaSPL21-6DHapII\&III [57], and TaSAP7-B (C) [58]. Moreover, the frequency of favorable allelic variation TaPPH-7A-1 (A) was increased from landraces (Population 2) to modern cultivars (Population 3) in Chinese ten wheat Zones. However, in Population 3, the maximum frequency of TaPPH-7A-1 (A) was only $63.6 \%$ in Zone VI (Additional file 4: Table S2, Fig. $6 a$ and b). Thus, there is a large potential for increasing TGW by selecting favourable allelic variation TaPPH-7A-1 (A) in high-yield wheat breeding.

\section{Conclusions}

The TaPPH-7A gene was cloned. Four SNPs and one 2bp InDel were observed. A molecular marker TaPPH7A-dCAPS was developed based on a SNP at $1299 \mathrm{bp}$ (A/G). Favourable allelic variation TaPPH-7A-1 (A) was found to be associated with high TGW and Chl content, 
but not with SN and GN in Population 1. The frequency of favourable allelic variation TaPPH-7A-1 (A) was maintained a relatively stable level of about $56 \%$ from 1960 s to 1990 s in Population 3, thus the favourable allelic variation should be valuable, and could be selected to increase grain yield by improving the source (chlorophyll content) and sink (the developing grain) simultaneously. The newly developed molecular marker TaPPH$7 A$-dCAPS could be integrated into a breeding kit of screening high TGW wheat for marker-assisted selection.

\section{Methods}

\section{Plant materials}

Chang 4738, a wheat variety with a high TGW and a slowly chlorophyll degradation rate after anthesis, was used to clone TaPPH-7A gene. A wheat diversity panel (36 accessions, Additional file 6: Table S4) was chosen to detect polymorphisms in TaPPH-7A genomic sequence. Fifteen wheat species of different ploidy and a set of nullitetrasomic lines of Chinese Spring were used for chromosome location of TaPPH. A DH population [59], derived from a cross of Hanxuan $10 \times$ Lumai 14, consisting of 150 lines, was used for linkage mapping of TaPPH.

Three wheat germplasm populations were used as plant materials to analyze the allelic variation in TaPPH$7 A$. Population 1 consisted of 323 accessions (Additional file 3: Table S1), including 275 modern cultivars, 36 advanced lines, and 12 landraces, was used for association analysis of the target gene allelic variation and phenotypic traits. Population 2 (157 landraces) and Population 3 (348 modern cultivars) [35] (provided by Dr. Xueyong Zhang, at Chinese Academy of Agricultural Sciences) were used to analyze the frequency of favorable allelic variation in different wheat production zones and wheat breeding history, and to evaluate the utilization value of the newly developed molecular markers.

All wheat accessions were legally obtained from Chinese Crop Germplasm Resources Information System (http:// www.cgris.net/zhongzhidinggou/index.php).

\section{Field management and phenotypic assessment}

Population 1 was planted at Experiment Stations at Changping (116 $\left.13^{\prime} \mathrm{E} ; 40^{\circ} 13^{\prime} \mathrm{N}\right)$ and Shunyi (116 $56^{\prime} \mathrm{E}$; $40^{\circ} 23^{\prime} \mathrm{N}$ ) in Beijing over 3 growing seasons, i.e. in 2015 at Shunyi, 2016 at Changping and Shunyi, and 2017 at Shunyi. The experiment field was divided into two plots with different water regimes: rain-fed (drought stressed, DS) and well-watered (WW). The DS plots were not irrigated during the whole growing season but had rainfalls of $173 \mathrm{~mm}, 143 \mathrm{~mm}$, and $116 \mathrm{~mm}$, respectively. The WW plots were irrigated with $750 \mathrm{~m}^{3} / \mathrm{ha}(75 \mathrm{~mm})$ : before winter, at booting, flowering and grain filling when the amounts of rainfall were insufficient during each corresponding period. In addition, a heat stress experiment (HS) was conducted by adding polythene covers over the plots at Shunyi in 2015 and 2016. The other field managements, such as fertilization, disease and pest control were the same as local production conditions. Phenotypic assessment was performed under 12 environments (E1 to E12). E1 to E12 indicated the environments at Shunyi in 2015 under WW, DS, WW + HS and DS + HS, Changping in 2016 under WW and DS, Shunyi in 2016 under WW, DS, WW + HS and DS + HS, Shunyi in 2017 under WW and DS, respectively.

Yield-related traits, including spike number per plant $(\mathrm{SN})$, grain number per spike (GN) and TGW, were measured under 12 environments. Chl content (SPAD value) was tested with a handheld portable chlorophyll meter (SPAD-502, Konica-Minolta, Tokyo) at flowering and grain filling stages in two environments (E11 and E12) (Additional file 7: Table S4). The SN, GN, TGW and Chl content were measured using five plants, separately.

Population 3 was grown at Luoyang $\left(112^{\circ} 45^{\prime} \mathrm{E} ; 36^{\circ} 61^{\prime}\right.$ N) in Henan Province during the 2001-2002 and 20042005 , and Shunyi $\left(116^{\circ} 56^{\prime} \mathrm{E} ; 40^{\circ} 23^{\prime} \mathrm{N}\right)$ in Beijing during 2009-2010 cropping seasons [60]. TGW was measured in all the three environments.

\section{DNA and RNA extraction and TaPPH-A gene cloning}

Seedlings of Chang 4738 were used as experimental materials. Genomic DNA was extracted by the CTAB method [61]. Total RNA was extracted using an RNAprep Pure Plant Kit (Tiangen, Beijing) following the manufacturer's instructions. First-strand cDNA was synthesized with a FastQuant RT Kit (with gDNase) (Tiangen, Beijing).

The nucleotide sequence of the rice NYC3 (Os06 g0354700) gene published on China Rice Data Center (http://www.ricedata.cn/gene/index.htm) was used for a blast search against the reference sequence of Chinese Spring from URGI Blast website (https://urgi.versailles. inra.fr/blast/blast.php). All Chinese Spring wheat scaffold sequences with high similarity to OsNYC3 sequence were downloaded. According to the scaffold sequences, three pairs of 7A-specific primers were designed using the software Primer Premier 5.0. All primers were synthesized by Beijing Huada Genomics, and listed in Table 3. The first pair of 7A-specific primers, C-F/R, was used to amplify coding sequence of TaPPH-A. The second pair of 7A-specific primers, G-F/R, was used to amplify genomic sequence of TaPPH-A. The third pair of 7Aspecific primers, $\mathrm{P}-\mathrm{F} / \mathrm{R}$, was used to amplify the promoter sequence of TaPPH-A.

The cDNA and genomic DNA of Chang 4738 were used as templates. PCR amplification was performed in $20 \mu \mathrm{L}$ volume including $1 \mu \mathrm{L} 50 \mathrm{ng} / \mu \mathrm{L}$ cDNA or genomic DNA, $0.2 \mu \mathrm{M}$ of each primer, $0.2 \mathrm{mM}$ dNTPs, $4 \mu \mathrm{L} 5 \times$ TransStart $^{\circ}$ FastPfu Buffer, and $0.4 \mu \mathrm{L}(2.5 \mathrm{U} / \mu \mathrm{L})$ TransStart $^{\circ}$ 
Table 3 Primer sequences used in this study

\begin{tabular}{lll}
\hline Primer name & Primer sequence $\left(5^{\prime}\right.$-3') $^{\prime}$ & Function \\
\hline C-F & ATGGAATGGTTCTTCCAGTCAC & Cloning coding sequence \\
G-R & TCATCTGGATACTACCCGTATGC & \\
G-R & GGCACCAAGAATAGCAAGGC & Cloning TaPPH-A genomic specific sequence \\
P-F & TCATCTGGACACTACCTGTATGTGGAGG & \\
P-R & TGGTTCGCAGGGATGACTGTAAC & Cloning TaPPH-A promoter specific sequence \\
RT-F & CCATCGTCCACACCTTGTAATCA & \\
RT-R & TATTGGGGTATCAGAGTCAAGCAG & Expression analysis \\
RT-Actin-F & AATCATCTGGATACTACCCGTATGC & \\
RT-Actin-R & CTCCCTCACAACAACAACCGC & Endogenous control \\
ECoRI-F & TACCAGGAACTTCCATACCAAC & \\
ECoRI-R & AAGTCTTCGTTGGTCTCAC & Marker TaPPH-7A-dCAPS developed for SNP-1299 (A/G) \\
\hline
\end{tabular}

FastPfu DNA Polymerase (TransGen Biotech, Beijing). The amplification program consisted of an initial denaturation at $95^{\circ} \mathrm{C}$ for $5 \mathrm{~min}$, followed by 35 cycles of denaturation at $95^{\circ} \mathrm{C}$ for $30 \mathrm{~s}$, annealing at $58^{\circ} \mathrm{C}$ for $45 \mathrm{~s}$, and extension at $72^{\circ} \mathrm{C}$ for $1-2 \mathrm{~min}$, with a final extension at $72{ }^{\circ} \mathrm{C}$ for $15 \mathrm{~min}$. The PCR products were separated by electrophoresis in $1.2 \%$ agarose gel. The target bands were purified using Gel Extraction Kit (BIOMIGA, China). The purified PCR product was cloned into $p E A S Y^{\circ}$-Blunt cloning vector (TransGen Biotech, Beijing), then was transformed to $33 \mu \mathrm{L}$ Trans1-T1 Phage Resistant Chemically Competent Cells (TransGen Biotech, Beijing) by heat shock. Six positive clones of each wheat genotype were sequenced. The sequence alignment was performed using SeqMan (DNASTAR Lasergene 7.1.0). The gene structure of TaPPH-A was determined using MegAlign (DNASTAR Lasergene 7.1.0) through alignment the coding and genomic sequences.

\section{Molecular marker development}

The sequences of TaPPH-7A cloned from the wheat diversity panel were aligned by SeqMan for screening polymorphism. Molecular marker(s) were developed based on polymorphism sites. A dCAPS marker was designed using dCAPS Finder 2.0 (http://helix.wustl.edu/ dcaps/dcaps.html). The primer pair of dCAPS marker was EcoRI-F/R. Genotyping was performed by two rounds of PCR and one enzyme digestion following the procedure described by Miao, et al. [56].

\section{Population structure and association analysis}

Population 1 was performed on Wheat 660 K SNP Array, which consisted of 630,517 SNPs [62]. By removing nucleotide variations with missing rates $\geq 0.2$ and minor allele frequency $(<0.05), 395,681$ SNPs were eventually used to detect the structure of Population 1 by software STRUCTURE 2.3.4 [59]. A general linear model was performed in TASSEL 2.1 for analyzing significant associations between the target gene allelic variation and and phenotypic traits. Associations were considered significant at $P<0.05$. Statistical analysis was conducted by SPSS 19.0 software (SPSS Corp., Chicago, IL, USA).

\section{Analysis of gene expression with quantitative real-time PCR}

A primer pair RT-F/R (Table 3) was designed to use for analyzing the expression of TaPPH-7A in different wheat genotypes. Quantitative real-time PCR (qRT-PCR) was performed in triplicate with Roche LightCycler ${ }^{\oplus} 96$ using the SYBR ${ }^{\odot}$ Premix Ex Taq ${ }^{\mathrm{Tm}}$ (Tli RNaseH Plus) (Takara, Japan). The qRT-PCR reaction system with specific primer contains $10 \mu \mathrm{L} 2 \times$ SYBR Premix Ex Taq ${ }^{\mathrm{Tm}}, 0.4 \mu \mathrm{L}$ $50 \times$ Rox Reference Dye II, $0.4 \mu \mathrm{L}(5 \mu \mathrm{M})$ of each primer (Table 3), $1 \mu \mathrm{L}$ cDNA template, and $7.8 \mu \mathrm{L} \mathrm{ddH}_{2} \mathrm{O}$. The reaction procedure was as follows: denaturation at $95^{\circ} \mathrm{C}$ for $2 \mathrm{~min}$; followed by 45 cycles at $95^{\circ} \mathrm{C}$ for $20 \mathrm{~s}, 60^{\circ} \mathrm{C}$ for $20 \mathrm{~s}$, and $72{ }^{\circ} \mathrm{C}$ for $20 \mathrm{~s}$. TaActin was used as the endogenous control of normalizing expression levels of different samples. Gene relative expression levels were calculated using the $2^{-\triangle \Delta C T}$ method [63]. The statistical analysis of $\Delta \Delta C_{\mathrm{T}}$ according to the method described by Zhang et al. [35].

\section{Additional files}

Additional file 1: Figure S1. Frequencies of $\mathrm{TaPPH}-7 \mathrm{~A}$ allelic variation in Populations 1-3. (DOCX $16 \mathrm{~kb}$ )

Additional file 2: Figure S2. The melting curves of qRT-PCR for genes TaPPH-7A and TaActin. The blue and red lines indicate TaPPH-7A and TaACtin, respectively. (DOCX $60 \mathrm{~kb}$ )

Additional file 3: Table S1. The information of 12 wheat varieties for qRT-PCR analysis (DOCX $14 \mathrm{~kb}$ )

Additional file 4: Table S2. The frequencies (\%) of TaPPH-7A allelic variation in Populations 2 and 3 from ten Chinese wheat production zones (DOCX $16 \mathrm{~kb}$ ) 
Additional file 5: Table S3. The information of Population 1 and their genotypes of TaPPH-7A (DOCX $51 \mathrm{~kb})$

Additional file 6: Table S4. The information of the wheat diversity panel and their genotypes of TaPPH-7A (DOCX $28 \mathrm{~kb}$ )

Additional file 7 Table S5. Statistical analysis on traits of Population 1 (DOCX $28 \mathrm{~kb}$ )

\section{Abbreviations}

Chl: Chlorophyll; dCAPS: Derived cleaved amplified polymorphic sequence; DH: Doubled haploid; DS: Drought stress; GN: Grain number per spike; PPH: Pheophytin pheophorbide hydrolase; QTL: Quantitative trait loci; SN: Spike number per plant; SNP: Single nucleotide polymorphism; TGW: Thousand-grain weight; WW: Well-watered

\section{Acknowledgments}

We thank our colleagues Dr. Xueyong Zhang (Institute of Crop Science, Chinese Academy of Agricultural Sciences) for providing DNA samples of Population 2 (157 landraces) and Population 3 (348 modern cultivars).

\section{Authors' contributions}

DS and RJ designed the research and revised the manuscript. HW performed the experiments, analyzed the data, and drafted the manuscript. SW, XC, and $\mathrm{CH}$ helped to measure agronomic traits. All the authors read and approved the final manuscript.

\section{Funding}

This work was supported by the National Natural Science Foundation of China (31671607), the National Key R\&D Program of China

(2017YFD0300202), and National Science and Technology Major Projects for Cultivation of New Transgenic Varieties (2018ZX0800917B). The funding bodies provided only the experimental cost, including data collection, and publication fee for this study. However, the experimental design, data analysis and interpretation, and writing the manuscript were managed by the contributing authors.

\section{Availability of data and materials}

All data generated or analysed during this study are included in this published article [and its Additional files].

\section{Ethics approval and consent to participate}

Not applicable.

\section{Consent for publication}

Not applicable.

\section{Competing interests}

The authors declare that they have no competing interests.

\section{Received: 1 April 2019 Accepted: 20 June 2019}

Published online: 08 July 2019

\section{References}

1. Mayer KFX, Rogers J, Dolezel J, Pozniak C, Eversole K, Feuillet C, et al. A chromosome-based draft sequence of the hexaploid bread wheat (Triticum aestivum) genome. Science. 2014;345:1251788-98.

2. Su Z, Hao C, Wang L, Dong Y, Zhang X. Identification and development of a functional marker of TaGW2 associated with grain weight in bread wheat (Triticum aestivum L.). Theor Appl Genet. 2011;122:211-23.

3. Gupta PK, Rustgi S, Kumar N. Genetic and molecular basis of grain size and grain number and its relevance to grain productivity in higher plants. Genome. 2006;49:565-71.

4. Zheng TC, Zhang XK, Yin GH, Wang LN, Han YL, Chen L, et al. Genetic gains in grain yield, net photosynthesis and stomatal conductance achieved in Henan Province of China between 1981 and 2008. Field Crops Res. 2011; 122:225-33.

5. Borrell AK, van Oosterom EJ, Mullet JE, George-Jaeggli B, Jordan DR, Klein $P E$, et al. Stay-green alleles individually enhance grain yield in sorghum under drought by modifying canopy development and water uptake patterns. New Phytol. 2014;203:817-30.
6. Thomas H, Smart CM. Crops that stay green. Ann Appl Biol. 1993;123:193219.

7. Gregersen PL, Holm PB, Krupinska K. Leaf senescence and nutrient remobilisation in barley and wheat. Plant Biol. 2008;10:37-49.

8. Thomas H, Howarth CJ. Five ways to stay green. J Exp Bot. 2000;51:329-37.

9. Tian FX, Gong JF, Wang GP, Wang GK, Fan ZY, Wang W. Improved drought resistance in a wheat stay-green mutant tasg 1 under field conditions. Biol Plant. 2012;56:509-15.

10. Horie $\mathrm{Y}$, Ito $\mathrm{H}$, Kusaba M, Tanaka R, Tanaka A. Participation of chlorophyll $b$ reductase in the initial step of the degradation of light-harvesting chlorophyll a/b-protein complexes in Arabidopsis. J Biol Chem. 2009;284: 17449-56.

11. Harpaz Saad S, Azoulay T, Arazi T, Ben Yaakov E, Mett A, Shiboleth Y, et al. Chlorophyllase is a rate-limiting enzyme in chlorophyll catabolism and is posttranslationally regulated. Plant Cell. 2007;19:1007-22.

12. Adriana P, Gaby T, Iwona A, Maria R, Stefan HR. Chlorophyll breakdown: Pheophorbide a oxygenase is a Rieske-type iron-sulfur protein, encoded by the accelerated cell death 1 gene. P Natl Acad Sci USA. 2003;100:15259-64.

13. Shimoda Y, Ito H, Tanaka A. Arabidopsis STAY-GREEN, Mendel's green cotyledon gene, encodes magnesium-dechelatase. Plant Cell. 2016;28:2147-60.

14. Wu S, Li Z, Yang L, Xie Z, Chen J, Zhang W, et al. NON-YELLOWING2 (NYE2), a close paralog of NYE1, plays a positive role in chlorophyll degradation in Arabidopsis. Mol Plant. 2016;9:624-7.

15. Schelbert S, Aubry S, Burla B, Agne B, Kessler F, Krupinska K, et al. Pheophytin pheophorbide hydrolase (Pheophytinase) is involved in chlorophyll breakdown during leaf senescence in Arabidopsis. Plant Cell. 2009:21:767-85.

16. Morita R, Sato Y, Masuda Y, Nishimura M, Kusaba M. Defect in non-yellow coloring 3 , an $\alpha / \beta$ hydrolase-fold family protein, causes a stay-green phenotype during leaf senescence in rice. Plant J. 2009;59:940-52.

17. Zhang J, Yu GH, Wen WW, Ma XQ, Xu B, Huang BR. Functional characterization and hormonal regulation of the PHEOPHYTINASE gene LPPPH controlling leaf senescence in perennial ryegrass. J Exp Bot. 2016;67: 935-45.

18. Liu YN, He ZH, Appels R, Xia XC. Functional markers in wheat: current status and future prospects. Theor Appl Genet. 2012:125:1-10.

19. Kuchel H, Fox R, Reinheimer J, Mosionek L, Willey N, Bariana H, et al. The successful application of a marker-assisted wheat breeding strategy. Mol Breeding. 2007;20:295-308.

20. Useche FJ, Gao G, Harafey M, Rafalski A. High-throughput identification, database storage and analysis of SNPS in EST sequences. Genome Inform. 2001;12:194-203.

21. Tang JF, Vosman B, Voorrips RE, van der Linden CG, Leunissen JAM. QualitySNP: a pipeline for detecting single nucleotide polymorphisms and insertions/deletions in EST data from diploid and polyploid species. BMC Bioinformatics. 2006;7:438-52.

22. Collard BC, Mackill DJ. Marker-assisted selection: an approach for precision plant breeding in the twenty-first century. Philos T R Soc B. 2008:363:557-72.

23. Wang BY, Tan HW, Fang WP, Meinhardt LW, Mischke S, Matsumoto T, et al. Developing single nucleotide polymorphism (SNP) markers from transcriptome sequences for identification of longan (Dimocarpus longan) germplasm. Hortic Res. 2015;2:14065-74

24. Zhang $Y X$, Wang $\mathrm{LH}$, Xin HG, Li DH, Ma CX, Xia D, et al. Construction of a high-density genetic map for sesame based on large scale marker development by specific length amplified fragment (SLAF) sequencing. BMC Plant Biol. 2013;13:141.

25. Qi ZM, Huang L, Zhu RS, Xin DW, Liu CY, Han X, et al. A high-density genetic map for soybean based on specific length amplified fragment sequencing. PLoS One. 2014;9:e104871.

26. Törjék O, Berger D, Meyer RC, Müssig C, Schmid KJ, Rosleff ST, et al. Establishment of a high-efficiency SNP-based framework marker set for Arabidopsis. Plant J. 2003:36:122-40.

27. Nemri A, Atwell S, Tarone AM, Huang YS, Zhao K, Studholme DJ, et al. Genome-wide survey of Arabidopsis natural variation in downy mildew resistance using combined association and linkage mapping. Proc Natl Acad Sci U S A. 2010;107:10302-7.

28. Hayashi K, Hashimoto N, Daigen M, Ashikawa I. Development of PCR-based SNP markers for rice blast resistance genes at the Piz locus. Theor Appl Genet. 2004;108:1212-20.

29. Agrama HA, Eizenga GC, Yan W. Association mapping of yield and its components in rice cultivars. Mol Breeding. 2007;19:341-56. 
30. Guillet-Claude C, Birolleau-Touchard C, Manicacci D, Rogowsky PM, Rigau J, Murigneux A, et al. Nucleotide diversity of the ZmPox3 maize peroxidase gene: relationships between a MITE insertion in exon 2 and variation in forage maize digestibility. BMC Genet. 2004;5:19-29.

31. Thornsberry JM, Goodman MM, Doebley J, Kresovich S, Nielsen D. Dwarf8 polymorphisms associate with variation in flowering time. Nat Genet. 2001; 28:286-9.

32. Li Q, Lin L, Yang XH, Warburton ML, Bai GH, Dai JR, et al. Relationship, evolutionary fate and function of two maize co-orthologs of rice GW2 associated with kernel size and weight. BMC Plant Biol. 2010;10:143-57.

33. Li B, Li QR, Mao XG, Li A, Wang JY, Chang XP, et al. Two novel AP2/EREBP transcription factor genes TaPARG have pleiotropic functions on plant architecture and yield-related traits in common wheat. Front Plant Sci. 2016; 7:1191-203.

34. Jiang QY, Hou J, Hao CY, Wang LF, Ge HM, Dong YS, et al. The wheat (T. aestivum) sucrose synthase 2 gene (TaSus2) active in endosperm development is associated with yield traits. Funct Integr Genomic. 2011;11: 49-61.

35. Zhang B, Liu X, Xu WN, Chang JZ, Li A, Mao XG, et al. Novel function of a putative MOC1 ortholog associated with spikelet number per spike in common wheat. Sci Rep. 2015;5:12211.

36. Somers DJ, Kirkpatrick R, Moniwa M, Walsh A. Mining single-nucleotide polymorphisms from hexaploid wheat ESTs. Genome. 2003:46:431-7.

37. Ravel C, Praud S, Murigneux A, Canaguier A, Sapet F, Samson D, et al. Single-nucleotide polymorphism frequency in a set of selected lines of bread wheat (Triticum aestivum L.). Genome. 2006;49:1131-9.

38. Nasu S, Suzuki J, Ohta R, Hasegawa K, Yui R, Kitazawa N, et al. Search for and analysis of single nucleotide polymorphisms (SNPS) in rice (Oryza sativa, Oryza rufipogon) and establishment of SNP markers. DNA Res. 2002;9:163-71.

39. Yang Y, Zhao XL, Xia LQ, Chen XM, Xia XC, Yu Z, et al. Development and validation of a Viviparous-1 STS marker for pre-harvest sprouting tolerance in Chinese wheats. Theor Appl Genet. 2007;115:971-80.

40. Zhang L, Zhao YL, Gao LF, Zhao GY, Zhou RH, Zhang BS, et al. TaCKX6-D1, the ortholog of rice OsCKX2, is associated with grain weight in hexaploid wheat. New Phytol. 2012;195:574-84.

41. Zhu XF, Zhang HP, Hu MJ, Wu ZY, Jiang H, Cao JJ, et al. Cloning and characterization of Tabas 1-B1 gene associated with flag leaf chlorophyll content and thousand-grain weight and development of a gene-specific marker in wheat. Mol Breeding. 2016;36:142-53.

42. Sun YW, He ZH, Ma WJ, Xia XC. Alternative splicing in the coding region of Ppo-A1 directly influences the polyphenol oxidase activity in common wheat (Triticum aestivum L.). Funct Integr Genomics. 2011;11:85-93.

43. Xiao J, Li J, Yuan L, Tanksley SD. Identification of QTLs affecting traits of agronomic importance in a recombinant inbred population derived from a subspecific rice cross. Theor Appl Genet. 1996;92:230-44.

44. Shi S, Azam FI, Li H, Chang X, Li B, Jing R. Mapping QTL for stay-green and agronomic traits in wheat under diverse water regimes. Euphytica. 2017;213: 246-64

45. Yang $\mathrm{DL}$, Jing $\mathrm{RL}$, Chang XP, Li W. Quantitative trait loci mapping for chlorophyll fluorescence and associated traits in wheat (Triticum aestivum L. ). J Integr Plant Biol. 2007:49:646-54.

46. Bhusal N, Sharma P, Sareen S, Sarial AK. Mapping QTLs for chlorophyll content and chlorophyll fluorescence in wheat under heat stress. Biol Plant. 2018;62:721-31.

47. Ilyas M, llyas N, Arshad M, Kazi AG, Kazi AM, Waheed A. QTL mapping of wheat doubled haploids for chlorophyll content and chlorophyll fluorescence kinetics under drought stress imposed at anthesis stage. Pak J Bot. 2014;46:1889-97.

48. Wang LF, Ge HM, Hao CY, Dong YS, Zhang XY. Identifying loci influencing 1,000-kernel weight in wheat by microsatellite screening for evidence of selection during breeding. PLoS One. 2012;7:e29432

49. Groos C, Robert N, Bervas E, Charmet G. Genetic analysis of grain proteincontent, grain yield and thousand-kernel weight in bread wheat. Theor Appl Genet. 2003;106:1032-40.

50. Somers DJ, Isaac P, Edwards K. A high-density microsatellite consensus map for bread wheat (Triticum aestivum L.). Theor Appl Genet. 2004;109:1105-14.

51. Cuthbert JL, Somers DJ, Brûlé-Babel AL, Brown PD, Crow GH. Molecular mapping of quantitative trait loci for yield and yield components in spring wheat (Triticum aestivum L.). Theor Appl Genet. 2008;117:595-608.

52. Andersen JR, Lubberstedt T. Functional markers in plants. Trends Plant Sci. 2003;8:554-60.
53. Bagge $M$, Xia X, Lübberstedt T. Functional markers in wheat. Curr Opin Plant Biol. 2007;10:211-6.

54. Gill BS, Appels R, Bothaoberholster AM, Buell CR, Bennetzen JL, Chalhoub B, et al. A workshop report on wheat genome sequencing: international genome research on wheat consortium. Genetics. 2004;168:1087-96.

55. He ZH, Zhuang QS, Cheng SH, Yu ZW, Zhao ZD, Liu X. Wheat production and technology improvenment in China. J Agric For. 2018;8:107-14.

56. Miao LL, Mao XG, Wang JY, Liu ZC, Zhang B, Li WY, et al. Elite haplotypes of a protein kinase gene TaSnRK2.3 associated with important agronomic traits in common wheat. Front Plant Sci. 2017;08:368-78.

57. Zhang B, Xu WN, Liu X, Mao XG, Li A, Wang JY, et al. Functional conservation and divergence among homoeologs of TASPL20 and TASPL21, two SBP-box genes governing yield-related traits in hexaploid wheat. Plant Physiol. 2017; 174:1177-91.

58. Wang YX, Xu QF, Chang XP, Hao CY, Li RZ, Jing RL. A dCAPS marker developed from a stress associated protein gene TASAP7-B governing grain size and plant height in wheat. J Integr Agr. 2018;17:276-84.

59. Li L, Mao X, Wang J, Chang X, Reynolds M, Jing R. Genetic dissection of drought and heat-responsive agronomic traits in wheat. Plant Cell Environ. 2019. https://doi.org/10.1111/pce.13577.

60. Zheng J, Liu H, Wang YQ, Wang LF, Chang XP, Jing RL, et al. TEF-7A, a transcript elongation factor gene, influences yield-related traits in bread wheat (Triticum aestivum L.). J Exp Bot. 2014;65:5351-65.

61. Stein N, Herren G, Keller B. A new DNA extraction method for highthroughput marker analysis in a large-genome species such a Triticum aestivum. Plant Breed. 2001;120:354-6.

62. Li L, Peng Z, Mao X, Wang J, Chang X, Reynolds M, et al. Genome-wide association study reveals genomic regions controlling root and shoot traits at late growth stages in wheat. Ann Bot. 2019. https://doi.org/10.1093/aob/ mcz041.

63. Schmittgen TD, Livak KJ. Analyzing real-time PCR data by the comparative $\mathrm{C}_{\mathrm{T}}$ method. Nat Protoc. 2008;3:1101-8.

\section{Publisher's Note}

Springer Nature remains neutral with regard to jurisdictional claims in published maps and institutional affiliations.

Ready to submit your research? Choose BMC and benefit from:

- fast, convenient online submission

- thorough peer review by experienced researchers in your field

- rapid publication on acceptance

- support for research data, including large and complex data types

- gold Open Access which fosters wider collaboration and increased citations

- maximum visibility for your research: over $100 \mathrm{M}$ website views per year

At $\mathrm{BMC}$, research is always in progress.

Learn more biomedcentral.com/submissions 